\title{
Improved $\beta$-catenin detection in spinal cord tissue sections: autofluorescence quenching
}

\author{
Dauda Abdullahi ${ }^{1,3, *}$, Azlina Ahmad Annuar ${ }^{2}$ and Junedah Sanusi ${ }^{1}$ \\ ${ }^{1}$ Department of Anatomy, Faculty of Medicine, University of Malaya, Kuala Lumpur, Malaysia. \\ ${ }^{2}$ Department of Biomedical Science, Faculty of Medicine, University of Malaya, Kuala Lumpur, Malaysia. \\ ${ }^{3}$ Department of Anatomy, College of Medical Sciences, Abubakar Tafawa Balewa University Bauchi, Bauchi State, \\ Nigeria. \\ * Correspondence: daudageneticist@yahoo.com; Tel.: +2347064882193
}

Received: 15 April 2020; Accepted: 22 May 2020; Published: 29 May 2020

Edited by: Pike-See Cheah (Massachusetts General Hospital, Boston, USA)

Reviewed by: Mahmoud Bukar Maina (University of Sussex, UK); Usman Bala (Gombe State University, Nigeria) https://doi.org/10.31117/neuroscirn.v3i2.49

\begin{abstract}
Experimental studies on spinal cord regeneration are focusing on the windows of opportunity to improve spinal cord microenvironment via spinal-centric repair pathways. One pathway of particular interest is the $\mathrm{Wnt} / \beta$-catenin signalling pathway which plays a vital role in axonal guidance, synaptic assembly and function, neuronal survival and connectivity after spinal cord trauma to induce repair. Upregulation of $\beta$-catenin expression is often taken as evidence of regeneration mechanisms through the $\mathrm{Wnt} / \beta$-catenin pathway. However, these studies may not have optimised the staining protocol for $\beta$-catenin to enable accurate detection of the protein. Given possible issues with the background or endogenous tissue autofluorescence, there is a need to optimise the protocol further to allow better visualisation of $\beta$-catenin. So far, there are no studies which report optimising spinal cord tissues for $\beta$-catenin to reduce autofluorescence, and as $\beta$-catenin is widely used in spinal cord injury $(\mathrm{SCl})$ and other spinal cord tissue studies, thus it is an important issue to address. To achieve reliable detection and localisation of $\beta$-catenin, we utilised sequential quenching techniques using $1 \% \mathrm{NaBH}_{4}$ and $1 \mathrm{mM} \mathrm{CuSO}_{4}$ in $50 \mathrm{mM}$ ammonium acetate buffer to reduce both background and fixative-induced autofluorescence. Our results showed that sequential autofluorescence quenching is crucial in $\beta$-catenin detection, and this improved technique indicates that $\beta$-catenin is localised in the spinal cord white matter regions. Objective approach for the $\beta$-catenin localisation is highly significant as it unravelled an objective identification and illuminate the pattern of distribution of $\beta$-catenin for researcher focusing on spinal cord repair studies via the $\mathrm{Wnt} / \beta$-catenin pathway following $\mathrm{SCl}$.
\end{abstract}

Keywords: Spinal cord; $\beta$-catenin; autofluorescence quenching; regeneration

(C2020 by Abdullahi et al. for use and distribution in accord with the Creative Commons Attribution (CC BY-NC 4.0) license (https://creativecommons.org/licenses/by-nc/4.0/), which permits unrestricted non-commercial use, distribution, and reproduction in any medium, provided the original author and source are credited.

\subsection{INTRODUCTION}

In the light of identifying potential neuroregenerative protein which is involved in spinal cord repair, researchers are focusing on one of the most extensively studies pathway, $\mathrm{Wnt} / \beta$-catenin pathways, by utilising the power of fluorescence immunohistochemistry. However, due to known autofluorescence of neural tissues, the localisation, 
distribution, and pattern of expression of $\beta$-catenin may be hindered by the impostors which mimic the $\beta$ catenin expression.

Reliable detection and localisation of $\beta$-catenin are crucial as experimental studies have suggested this pathway is involved mediating spinal cord repair (Cuzzocrea et al., 2006; Dill et al., 2008; Liu et al., 2008; Suh et al., 2011; Tural Emon et al., 2017; Yin et al., 2008). Wnt (Wingless) proteins are a family of secreted glycoproteins (van Amerongen et al., 2008) that play key roles during the development of the nervous system, influencing cell proliferation and patterning, cell polarity and motility, axonal guidance, synaptic assembly and function, neuronal survival and connectivity and cell-cell adhesion (Ahmad-Annuar et al., 2006; Ciani \& Salinas, 2005; Curinga \& Smith, 2008). For example, previous studies revealed that the $\mathrm{Wnt} / \beta$-catenin plays significant roles in spinal cord injury (SCl) repair process (Cuzzocrea et al., 2006; Dill et al., 2008; Liu et al., 2008; Suh et al., 2011; Tural Emon et al., 2017; Yin et al., 2008). It has been shown that after $\mathrm{SCl}$, the $\mathrm{Wnt} / \beta$-catenin signalling pathway is activated. First, $\beta$-catenin dissociates from the Axin/APC/GSK-3 $\beta$ destruction complexes when the cytoplasmic segments of the Fz receptor interact with the low-density lipoprotein receptor-related protein $5 / 6$ (LRP5/6), inhibiting the activity of GSK-3 $\beta$ via Dvl, which leads to $\beta$-catenin stabilisation in the cytosol, and ultimately in the nucleus (Cuzzocrea et al., 2006; Dill et al., 2008; Liu et al., 2008; Suh et al., 2011; Tural Emon et al., 2017; Yin et al., 2008). Nuclear $\beta$-catenin combines with T-cell factor/Lymphoid enhancer factor (TCF/LEF) family of DNA-binding proteins to activate the expression of target genes, ultimately promoting axonal regeneration and accelerating the nerve function recovery process (Gao et al., 2016; Gao et al., 2015; Lu et al., 2016; Shen et al., 2017).

Despite the promising results correlating $\beta$-catenin to recovery after $\mathrm{SCl}$, there are possibilities of autofluorescence in the spinal cord tissue sections which could hinder convincing tissue interpretation concerning the expression of the $\beta$-catenin. Therefore, improving the detection of $\beta$-catenin through appropriate autofluorescence quenching would add credibility to data obtained in $\mathrm{SCl}$ repair studies. In this light, we set out an experiment where we observed autofluorescence and developed autofluorescence quenching techniques in the spinal cord to achieve reliable data.
The interpretation of fluorescently labelled $\beta$-catenin spinal cord tissue needs to take into account the presence of fixative-induced autofluorescence and also autofluorescent pigment lipofuscin and other tissue components such as collagen and elastin. Autofluorescence results from a natural or fixativeinduced emission of light by biological tissues when they have absorbed light, which is used to distinguish the light emitting from fluorescent markers (Davis et al., 2014; Kiernan \& Wessendorf, 2001; Monici, 2005). It has been reported that the presence of aldehyde fixatives and lipofuscin in nerve tissue can complicate the use of fluorescent immunohistochemical techniques because it fluoresces intensely under microscope filter systems (Schnell et al., 1999; $\underline{\text { Spitzer }}$ et al., 2011). It is, therefore, difficult to distinguish specific tissue fluorescence signals of protein of interest from that of false autofluorescence signals.

Considering the numerous attentions gained by $\beta$ catenin in spinal cord repair processes and the realisation of difficulties presented by possible false negative staining, we developed quenching methods that eliminate or reduce autofluorescence when looking at $\beta$-catenin in spinal cord regeneration studies. To date, no study has addressed these issues and may have potentially overlooked the contribution of autofluorescence in their studies.

\subsection{MATERIALS AND METHODS}

\subsection{Conventional approach to B-catenin detection in tissue}

The Faculty of Medicine Institutional Animal Care and Use Committee (FOM IACUC), University of Malaya, Kuala Lumpur, Malaysia, approved the surgical protocol used in this study with the reference number 2015-181103/ANAT/R/DA. Sprague Dawley (SD) rats were obtained from the Animal Unit of Experimental House, University of Malaya, Kuala Lumpur Malaysia. Eight weeks old male and female Sprague-Dawley rats were used in this study $(250 \pm 50 \mathrm{~g})$. The rats were kept at the standard conditions of temperature $\left(23 \pm 2^{\circ} \mathrm{C}\right)$ and humidity $(50 \pm 10 \%)$ with an alternating 12 -hour light/dark cycle. The $\beta$-catenin staining techniques were repeated three times for reliable and improved detection.

Published studies using $\beta$-catenin were used as an initial guide for the immunofluorescence. These studies were performed to detect $\beta$-catenin expression in intestinal tissue (Barker \& Born, 2008), 
foetal spinal cord (Ma et al., 2015) and adult rat spinal cord (Tural Emon et al., 2017). The animals were deeply anaesthetised and euthanised with a mixture of $0.35 \mathrm{~mL}$ of Ketamil (100 mg $/ \mathrm{mL}$ ketamine, Australia) and $0.15 \mathrm{~mL}$ of Xylazil $(20 \mathrm{mg} / \mathrm{mL}$ xylazine, Australia). Dissection was done on ice, freshly exposed and dissected spinal cord at the level of the twelfth thoracic vertebra (T12) were rapidly fixed by transferring to $4 \%$ paraformaldehyde in phosphatebuffered saline (PBS) overnight for immunofluorescence studies. The spinal cord tissues were inserted into tissue cassettes and processed using automatic tissue processor (Shandon Citadel 2000, Thermo Scientific) for a 12-hour period where tissues were processed through graded ethanol solutions, followed by clearing in xylene and finally embedded in paraffin, Table 1. In those earlier published studies on the $\beta$-catenin staining (Barker \& Born, 2008; Ma et al., 2015; Tural Emon et al., 2017), the tissues were not processed using whole animal perfusion, and there was no quenching of autofluorescence signals.

Table 1. Routine tissue processing (light microscopic) protocol

\begin{tabular}{ccc}
\hline Processes & Reagents & Duration \\
\hline Dehydration & $50 \%$ Ethanol & 1 hour \\
& $70 \%$ Ethanol & 1 hour \\
& $80 \%$ Ethanol & 1 hour \\
& $95 \%$ Ethanol & 1 hour \\
& $95 \%$ Ethanol & 1 hour \\
& Absolute Ethanol I & 1 hour \\
& Absolute Ethanol II & 1 hour \\
\hline Clearing & Xylene I & 1 hour \\
& Xylene II & 1 hour \\
& Xylene III & 1 hour \\
\hline Impregnation & Paraffin Wax I & 1 hour \\
& Paraffin Wax II & 1 hour \\
\hline Embedding at $\mathbf{5 6 ^ { \circ } \mathbf { C }}$ & Paraffin Wax \\
\hline
\end{tabular}

Serial spinal cord sections were cut from paraffin blocks on a rotary microtome and mounted on Poly-LLysine coated Superfrost glass slides for subsequent deparaffinisation and rehydration (Table 2). After antigen retrieval, sections were blocked with normal serum and incubated overnight at $4^{\circ} \mathrm{C}$ with the primary antibody, anti- $\beta$-catenin (Cell Signalling Technology). Sections were then washed in phosphate-buffered saline with Tween 20 (PBST) and incubated for 1 hour at room temperature in the secondary antibody (Alexa
Fluor 594). Processed tissues were counterstained using 4',6-diamidino-2-phenylindole (DAPI) and imaged for analysis.

\subsection{Improved staining technique and autofluorescence quenching in spinal cord sections}

With modifications, the previously described autofluorescence quenching techniques (Clancy \& Cauller, 1998; Kiernan \& Wessendorf, 2001; Schnell et al., 1999; Spitzer et al., 2011) were utilised to label $\beta$ catenin in the spinal cord of animals using antibodies against $\beta$-catenin ( $\beta$-catenin (D10A8) XP Rabbit mAB, Cell Signalling Technology) in both perfused and unperfused spinal cord. Contrary to other studies, transcardial hypoperfusion was performed using normal saline and 4\% paraformaldehyde in PBS with the aid of Minipuls 2 Perfusion Pump (Gilson). After deeply anaesthetising the rats, they were transcardially perfused using previously described methods (Gage et al., 2012). Tissues were then processed for frozen sections and formalin-fixed paraffin-embedded (FFPE) sections using a cryostat and rotary microtome, respectively.

\subsection{Quenching the autofluorescence caused by aldehyde fixative}

Frozen $14 \mu \mathrm{m}$ sections were directly washed three times in PBS. While the FFPE $7 \mu \mathrm{m}$ sections were initially deparaffinised and hydrated before PBS washing, as shown in Table 2. The frozen sections were made thicker than FFPE sections due to operation difficulty in block sectioning to maintain spinal tissue morphology. With modifications, tissue sections were then incubated in freshly prepared $1 \% \mathrm{NaBH}_{4}$ in PBS for $20 \mathrm{~min}$ followed by blocking in $1 \mathrm{XPBS} / 5 \%$ goat serum/0.3\% Triton for $60 \mathrm{~min}$ at room temperature as previously described (Clancy \& Cauller, 1998; Spitzer et al., 2011). During our experimental optimisation, we tested a range of concentrations and exposure times for the $\mathrm{NaBH}_{4}$ step, as described in the original paper (Clancy \& Cauller, 1998). Our modification was aimed at choosing the parameters that gave the best reduction of autofluorescence and background fluorescence without compromising the brightness and clarity of the $\beta$-catenin immunohistochemical signals. Specifically, sections were processed as shown in Table 2. However, for the FFPE, prior to blocking, heatinduced epitome (antigen) retrieval was performed followed by washing as shown in Table 2 . Subsequent immunostaining of $\beta$-catenin (Cell Signalling Technology) were performed according to the manufacturer's instruction where the sections were 
Table 2. Optimised procedure for immunofluorescence staining $\beta$-catenin.

\begin{tabular}{|c|c|c|}
\hline Steps & Reagents & Duration \\
\hline Deparaffinize & $\begin{array}{l}\text { Xylene I } \\
\text { Xylene II }\end{array}$ & $\begin{array}{l}5 \text { minutes } \\
5 \text { minutes }\end{array}$ \\
\hline Hydrate section to water & $\begin{array}{c}\text { Absolute Ethanol I } \\
\text { Absolute Ethanol II } \\
95 \% \text { Ethanol } \\
70 \% \text { Ethanol } \\
50 \% \text { Ethanol } \\
\mathrm{DH}_{2} \mathrm{O} \\
\end{array}$ & $\begin{array}{c}10 \text { minutes } \\
10 \text { minutes } \\
10 \text { minutes } \\
5 \text { minutes } \\
5 \text { minutes } \\
5 \text { minutes } \\
\end{array}$ \\
\hline Wash sections & PBS & $3 \times 5$ min each \\
\hline $1^{\text {st }}$ Autofluorescence Quenching & $\mathrm{NaBH}_{4}$ in PBS & 20 minutes \\
\hline $\begin{array}{l}\text { antigen retrieval treatment in microwave oven for the FFPE } \\
\text { sections }\end{array}$ & Citrate Buffer & $4 \mathrm{X}, 5 \mathrm{~min}$ each \\
\hline Cool slides & Citrate Buffer & \\
\hline Wash slides & $\mathrm{DH}_{2} \mathrm{O}$ & $3 X, 5$ min each \\
\hline Rinse slides & PBS & $3 \times 5$ min each \\
\hline Wipe off excess washing buffer and incubate sections & Normal Serum & 1 hour \\
\hline Blot off excess normal serum and incubate. & $1^{\circ}$ antibody & 1 hour \\
\hline Wash slides & PBS & $5 \mathrm{~min}$ \\
\hline Incubate sections in dark humidity chamber. & $2^{\circ}$ antibody & 1 hour \\
\hline Rinse slides in the dark. & PBST & $3 \times 5 \min$ each \\
\hline Rinse section & $\mathrm{DH}_{2} \mathrm{O}$ & 1 minute \\
\hline
\end{tabular}

blocked by normal goat serum and incubated overnight at $4^{\circ} \mathrm{C}$ with the primary antibody (anti- $\beta$-catenin). Sections were then washed in PBS and incubated for 1 hour at room temperature in the secondary antibody (Alexa Fluor 594).

\subsection{Quenching the autofluorescence caused by lipofuscin}

After immunofluorescence staining, tissue sections were washed three times in PBS, rinsed briefly in distilled water, and treated for $1 \mathrm{hr}$ in $1 \mathrm{mM} \mathrm{CuSO}_{4}$ in $50 \mathrm{mM}$ ammonium acetate, $\mathrm{pH} 5.0$ as previously

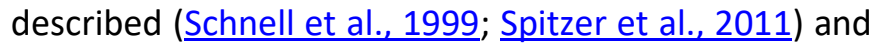
followed by another brief rinsing in distilled $\mathrm{H}_{2} \mathrm{O}$. Finally, processed tissues were counterstained using DAPI and incubated overnight at room temperature prior to image examination and analysis.

\subsection{RESULTS}

\subsection{Autofluorescence of spinal cord tissues is a technical issue in immunohistochemistry}

Using published protocols, first attempts to detect $\beta$ catenin was hampered by unexpected issues with autofluorescence of the spinal cord sections. Using the tissue preparation protocol as outlined in the conventional approach under the methodology section, tissues were labelled with the $\beta$-catenin primary antibody and the Alexa-Fluor 594 secondary antibody, and was excited under the 594 wavelengths and captured through the $\mathrm{Cy} 3$ channel. Significant autofluorescence was detected at the $515 \mathrm{~nm}$ wavelength, while FITC is usually excited around $488 \mathrm{~nm}$. Apparent false-positive signals were diffused throughout the entire spinal cord grey and white matter regions with significant overlap of stainings between $\beta$-catenin (red) and DAPI (blue) and FITC (green) (Figure $1 \mathrm{~A}-\mathrm{C}$ ).

This significant overlap seen in the $515 \mathrm{~nm}$ and Cy3 channels indicated that the $\beta$-catenin staining visualised may not be accurate. This overlap may be due to a slight overlap in the excitation range to excite the Cy3 and in the FITC channel (Figure 1D). Narrowing the excitation range of the fluorophores to eliminate any overlap did not solve the issue indicating that there might be an issue of autofluorescence.

The observed autofluorescence was later confirmed in spinal cord tissue sections which were only incubated in $1 \mathrm{X} P B S / 5 \%$ goat serum $/ 0.3 \%$ Triton and washed in PBS. The antibodies ( $\beta$-catenin and the Alexa-Fluor 594) incubation step was skipped before mounting in DAPI. Upon imaging, the sections showed endogenous fluorescence signals for DAP1 (Figure 1F). However, autofluorescence signals in the green channel were detected (Figure 1G) and were suspected to be 

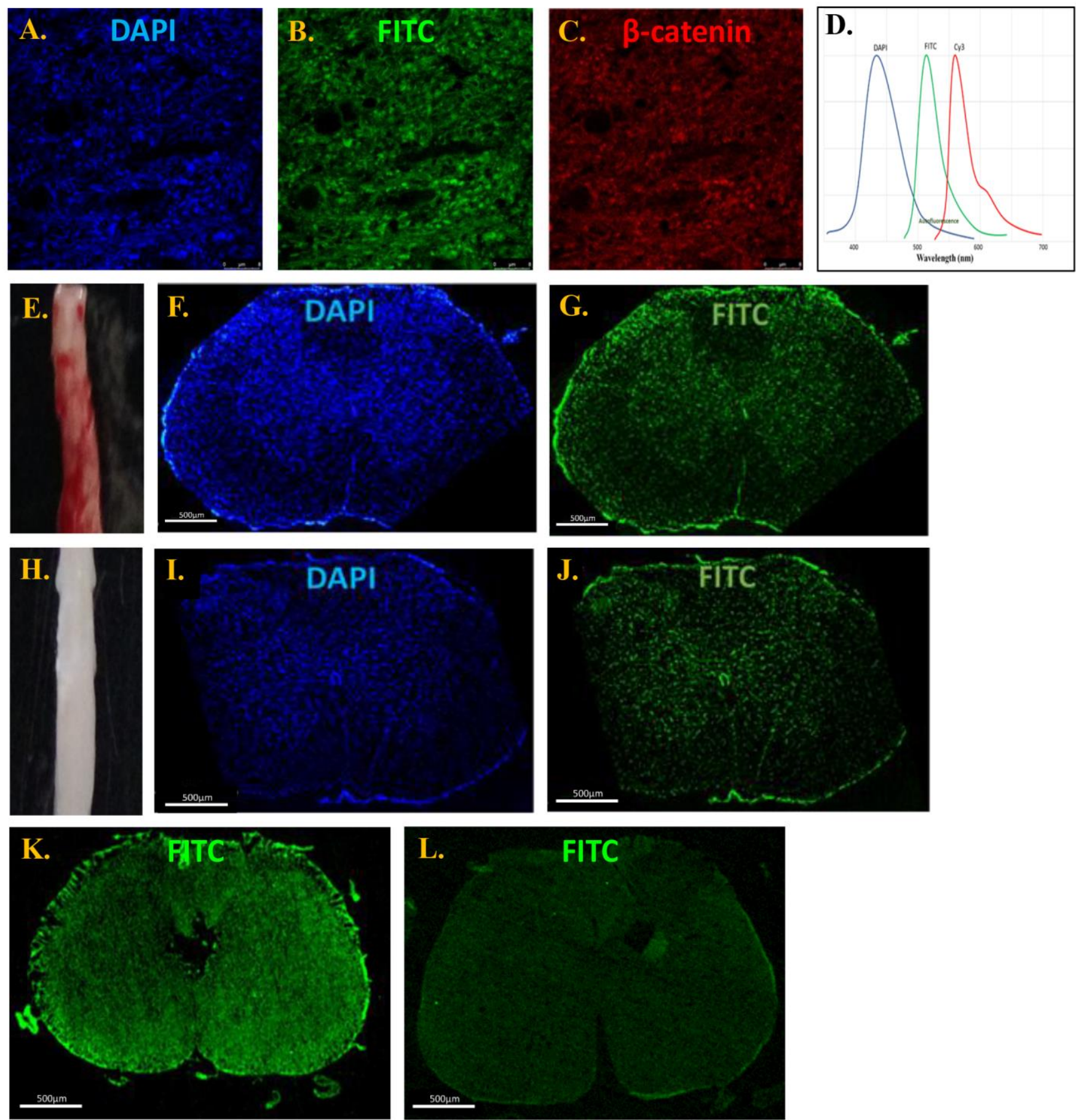

Figure 1. Quenching of autofluorescence signals in spinal cord tissue sections. Micrographs are showing the nuclear DAPI staining (A), autofluorescence signal (B) and $\beta$-catenin expression of the spinal cord (C), schematic emission and excitation spectra of three fluorescence channels (DAPI, FITC \& Cy3) showing the overlap of wavelengths between the FITC and Cy3 leading to false signals in the Cy3 channel which is not specific for $\beta$-catenin (D), longitudinal view of the gross anatomical spinal thoracic segment of the unperfused $(\mathbf{E})$ and transcardially hypoperfused rats $(\mathbf{H})$. Unperfused spinal cord exhibits more prominent green autofluorescent signals (G) than that in the perfused spinal cord $(J)$, and their corresponding sections are counterstained with DAPI (F \& I). When both treated with $\mathrm{NaBH}_{4}-\mathrm{CuSO}_{4}$, perfused (L) spinal cord exhibits reduced autofluorescence signal as compared to the unperfused (K) spinal section. 
lipofuscin and other autofluorescence structures in the unperfused rat spinal cord. The findings suggested that autofluorescence was a technical issue and needed further sequential procedures to eliminate it.

\subsection{Quenching the autofluorescence by perfusion reduced the background autofluorescence signals} As autofluorescence signals were prominent in the unperfused spinal cord tissues, preliminary optimisation was performed in an attempt to quench the autofluorescence by transcardial hypoperfusion to clear blood from the spinal cord (Figure 1E). It is wellknown that blood contributes to autofluorescence in tissues (Monici, 2005). After perfusion, the spinal cord tissue was cleared of blood (Figure $1 \mathrm{H}$ ). Subsequent microscopy of spinal cord sections without $\beta$-catenin primary or the Alexa Fluor 594 antibodies (1X PBS/5\% goat serum $/ 0.3 \%$ Triton $X-100)$, showed that the autofluorescence signals were still detected but had become less prominent (Figure $1 \mathrm{l}-\mathrm{J}$ ). This shows that perfusion is crucial to minimise autofluorescence in spinal cord tissue, but there was still a degree of persistent autofluorescence.

Further optimisation was needed before $\beta$-catenin could be reliably detected in the spinal cord tissue. Several other factors may contribute to the autofluorescence in tissues. For example, aldehyde, lipofuscin, blood vessels, elastin and collagen are within the group of biological components that cause autofluorescence and may lead to false-positive signals.
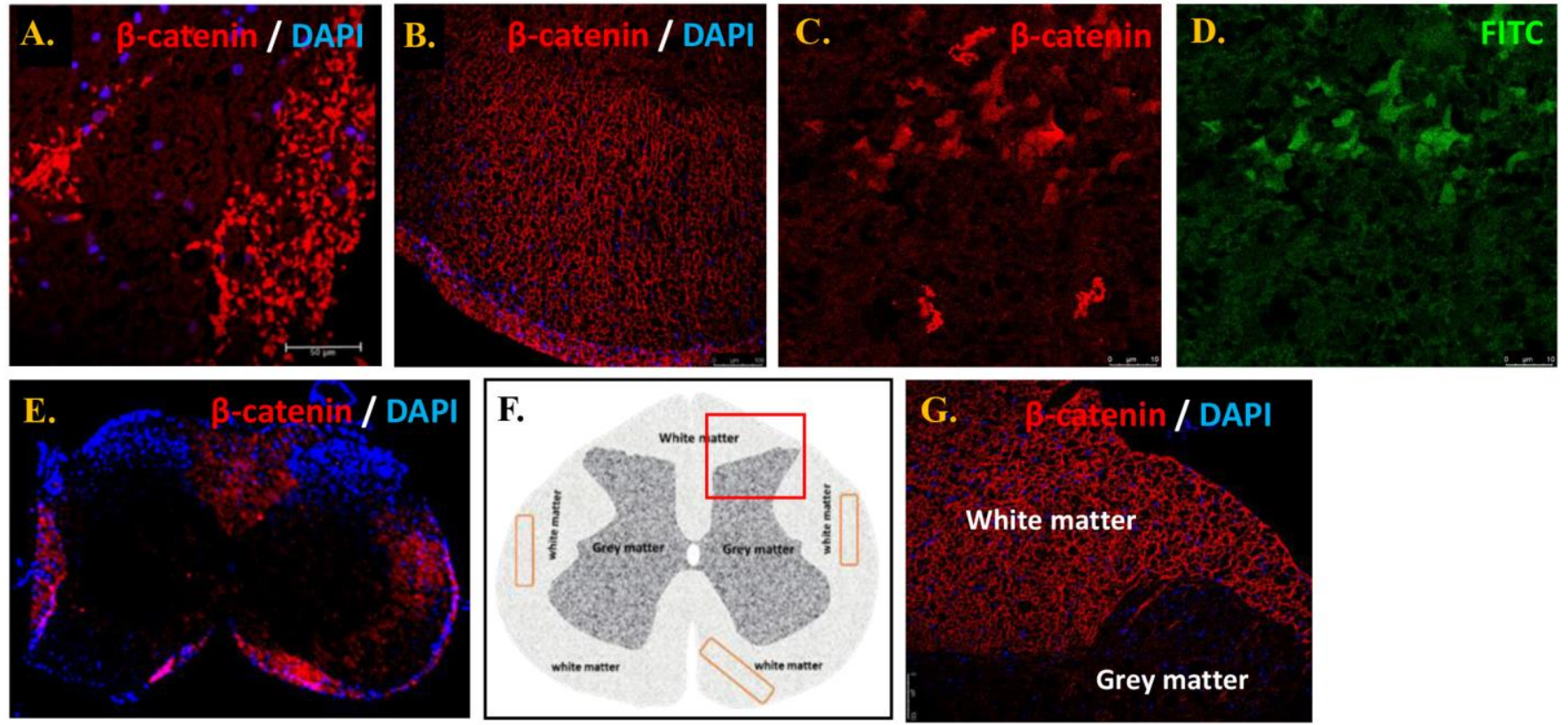

Figure 2. $\beta$-catenin signal detection in the perfused and $\mathrm{NaBH}_{4}-\mathrm{CUSO}_{4}$ treated spinal cord sections. Micrographs are showing clumps of irregular mass associated with $\beta$-catenin in unperfused and unquenched tissue section (A), autofluorescence signals mimicking expression of $\beta$-catenin signals upon optical zooming (C), same view of a section (in "C") depicting prominent green autofluorescent signals believed to be lipofuscin (D), regular $\beta$-catenin expression in transcardially perfused and autofluorescence quenched tissue sections (B), where the expression and localisation of $\beta$-catenin are evident in the white matter region of the spinal cord $(E)$, schematic illustration of the spinal cord where the orange boxes are areas of the $\beta$ catenin localisation in the white matter (F), and a confocal image at higher magnification of the area under the red square box in " $F$ " reveals clear expression and distribution of $\beta$-catenin in the white matter region of the spinal cord with lack of expression in the grey matter region at X63 objective (G).

\subsection{Quenching with $\mathrm{NaBH}_{4}$ and $\mathrm{CuSO}_{4}$ reduced the autofluorescence signals}

To further quench the autofluorescence signals, another step was added including treatment with $1 \%$ $\mathrm{NaBH}_{4}$ in PBS and $1 \mathrm{mM} \mathrm{CuSO}_{4}$ in ammonium acetate buffer to quench the lipofuscin autofluorescence.
$\mathrm{NaBH}_{4}$ is a chemical known to neutralise Schiff's bases through reduction of amine-aldehyde (such as formaldehyde) compounds into non-fluorescent salts (Clancy \& Cauller, 1998). $\mathrm{CuSO}_{4}$ composes of the $\mathrm{Cu}^{2+}$ and $\mathrm{SO}_{4}{ }^{2-}$ ions. $\mathrm{Cu}^{2+}$ was found to be able to quench lipofuscin (Schnell et al., 1999) using the protocol 
outlined in Table 2. Treatment with these chemicals significantly reduced the autofluorescence signals in the perfused spinal cord sections compared to the unperfused and untreated sections (Figure 1K-L).

\section{4 $\beta$-catenin signal detection in the perfused, and $\mathrm{NaBH}_{4}-\mathrm{CUSO}_{4}$ treated spinal cord sections}

Successful quenching of autofluorescence signals was achieved with perfusion and $\mathrm{NaBH}_{4}-\mathrm{CuSO}_{4}$ treatment. Thus, the next step was to determine if the expression of $\beta$-catenin could be more reliably detected as shown in the optimised protocol in Table 2. Some low levels of autofluorescence signals could still be detected, especially when viewed at higher magnification (63X) (Figure 2A-B). In sections that were not perfused or quenched, there was irregular staining of $\beta$-catenin together with stains of irregular structures (Figure 2A). Comparatively, in perfused and quenched sections, $\beta$ catenin expression was evenly distributed across the white matter regions and appeared as uniformly regular shaped, punctate structures. (Figure 2B).

The above-suspected autofluorescence in Figure 2A above in the conventional protocol was further checked and confirmed by optical zooming of some regions showing expression (Figure $2 \mathrm{C}$ ) and image was again captured in the same region of interest (ROI) at $515 \mathrm{~nm}$ wavelength (Figure 2D) which confirmed real autofluorescence signals believed to be lipofuscin mimics $\beta$-catenin expression in the unperfused rats. As shown in Figure 2, the specific labelling of $\beta$-catenin was questionable in unperfused, non- $\mathrm{NaBH}_{4}-\mathrm{CuSO}_{4}$ treated sections as a significant degree of signal overlaps were seen in the TRITC (red) channel (wavelength $594 \mathrm{~nm}$ ) where the $\beta$-catenin signal was expected. Besides, the FITC (green) channel (wavelength $488 \mathrm{~nm}$ ), which depicts an irregular mass of structures believed to be lipofuscin when compared to previously published data (Schnell et al., 1999).

The result indicated that spinal cord sections for $\beta$ catenin detection were best achieved by transcardial hypoperfusion prior to the systematic quenching steps with $\mathrm{NaBH}_{4}$ and $\mathrm{CuSO}_{4}$ to reduce the autofluorescence. This allows for more accurate and reliable $\beta$-catenin detection. Thus, transcardial hypoperfusion and quenching are necessary for reliable detection of $\beta$ catenin. Images were analysed to determine the pattern of expression of $\beta$-catenin expression in spinal cord tissues. $\beta$-catenin was found in the white matter regions at the level of T12 (Figure $2 \mathrm{E}$ ) indicated by the orange boxes in the schematic diagram (Figure 2F). Figure $2 \mathrm{G}$ is at a higher magnification (63X), which confirmed that the $\beta$-catenin is expressed in the white matter and not in the grey matter region.

\subsection{DISCUSSION}

Spinal cord injury $(\mathrm{SCl})$, including primary and secondary injury, is an unresolved challenge in experimental medical research. Secondary injury plays a critical role in $\mathrm{SCl}$ and is induced by various factors, such as apoptosis, oxidative stress, and the inflammatory immune response (Lu et al., 2016). It has been observed that when the $\mathrm{Wnt} / \beta$-catenin signalling pathway is activated, nerve cell apoptosis is inhibited; when the $W n t / \beta$-catenin signalling pathways are suppressed, the nerve cell apoptosis levels increased significantly (Gao \& Zhang, 2018). After SCl, the Wnt/ $\beta$ catenin signalling pathway is activated, $\beta$-catenin dissociates from the APC/Axin/GSK-3 $\beta$ complexes and receives upstream molecular signals. The protein then transports the signals to the nucleus to activate the target gene, ultimately promoting axonal regeneration and accelerating the nerve function recovery process (Gao et al., 2016; Gao et al., 2015; Gao \& Zhang, 2018; Lu et al., 2016; Shen et al., 2017) via the Wnt/ $\beta$-catenin signalling. All these recent studies have identified the molecular mechanisms in $\mathrm{SCl}$ repair process via the $\mathrm{Wnt} / \beta$-catenin signalling. As part of an ongoing study into the effect of natural products on $\mathrm{SCl}$, the involvement of $\beta$-catenin was studied. Unfortunately, the published protocols lead to unconvincing $\beta$-catenin staining in the spinal cord sections. Correct staining of $\beta$-catenin is essential to uncover its actual role. Thus, the protocol to stain $\beta$-catenin was developed to overcome the autofluorescence issue.

Autofluorescence has been considered to be a natural or fixative-induced emission of light by biological tissues when they have absorbed light and is used to distinguish the light emitting from fluorescent markers (Davis et al., 2014; Kiernan \& Wessendorf, 2001; Monici, 2005). Generally, tissue autofluorescence could be caused by lipofuscin (prominent in neurons, glial cells and a wide range of post-mitotic cells) (Kiernan \& Wessendorf, 2001), elastin \& collagen (typically from the wall of blood vessels) (Deyl et al., 1980; Kiernan \& Wessendorf, 2001; Monici, 2005).

Crucially, autofluorescence was not considered in the papers where the original protocols for immunofluorescence were taken from (Barker \& Born, 2008; Ma et al., 2015; Tural Emon et al., 2017; Zhang et al., 2013). In most of these studies, the tissues were neither refused nor quenched of autofluorescence signals (Barker \& Born, 2008; Ma et al., 2015; Tural 
Emon et al., 2017). Of these, one study performed perfusion but not $\mathrm{NaBH}_{4}-\mathrm{CuSO}_{4}$ tissue treatment to quench the spinal cord autofluorescence (Zhang et al., 2013) while detecting for $\beta$-catenin in spinal cord section.

We have reasons to believe that the photomicrograph by Zhang et al. (2013) might not be depicting the real expression of $\beta$-catenin because in the present study (Figure 2D), shows that these signals are likely to be the background and endogenous autofluorescence. In addition, Zhang and colleagues (2013) used a FITC conjugated secondary antibody, which is within the excitation range of autofluorescence (Spitzer et al., 2011). Careful selection of a secondary antibody (such as Alexa Fluor 594 as used in this study) is critical in fluorescently labelled neural tissue to reduce the risk of detecting autofluorescent signals. As the autofluorescence signals associated with the $\beta$-catenin expression remained after perfusion, it was clear that perfusion alone was not enough to reduce the autofluorescence signals.

We initially approached the quenching techniques using both the frozen and FFPE sections. However, after performing appropriate antigen retrieval on the FFPE sections, we observed no difference in the signal intensity of our protein of interest compared to frozen sections on the autofluorescence quenching outcome. Thus, images from FFPE sections were ultimately utilised for this study, considering the better preservation of tissue morphology in the FFPE section compared to frozen sections.

Several treatment options have been employed to reduce lipofuscin and fixative-induced autofluorescence in tissues. For example, Sudan Black treatment could be used to reduce lipofuscin fluorescence but not the emission signal of FITC and Alexa Fluor 594 (Kiernan \& Wessendorf, 2001; Romiin et al., 1999). Commercially available lipofuscin autofluorescence quencher TrueBlack ${ }^{\mathrm{TM}}$ appear to be effective in quenching but could reduce the signal intensity of protein of interest (Sun \& Chakrabartty, 2016). Whereas trypan blue treatment could be employed to reduce fixation-induced fluorescence, but this technique may not be suitable for multi-label experiments (Kiernan \& Wessendorf, 2001; Mosiman et al., 1997). Unlike the previous treatment options, our borate $\left(1 \% \mathrm{NaBH}_{4}\right)$ and cupric $\left(1 \mathrm{mM} \mathrm{CuSO}_{4}\right.$ in $50 \mathrm{mM}$ ammonium acetate) treatment protocol did not affect probe fluorescence intensity while it effectively reduced lipofuscin

and

fixative-induced

autofluorescence.

$\mathrm{NaBH}_{4}$ and $\mathrm{CuSO}_{4}$ in $50 \mathrm{mM}$ ammonium acetate buffer, have been used in attempts to reduce autofluorescence in brain sections (Clancy \& Cauller, 1998), and to eliminate lipofuscin autofluorescence in neural tissue sections (Schnell et al., 1999), respectively. Spitzer and colleagues reviewed and made a systematic comparison of earlier studies (Clancy \& Cauller, 1998; Schnell et al., 1999) on quenching false-positive signals in the brain tissue (Spitzer et al., 2011). Their study intended to look at the expression/localisation of serotonin (5-HT), glial fibrillary acidic protein (GFAP), $\beta$-tubulin III and $\mu$ opioid receptors, in the rat brain tissue. Based on the published protocols, tissue sections were treated with both $1 \% \mathrm{NaBH}_{4}$ and $1 \mathrm{mM} \mathrm{CuSO}_{4}$ in $50 \mathrm{mM}$ ammonium acetate buffer, and the approach successfully reduced background and cellular autofluorescence on perfused sections (Spitzer et al., 2011).

In this study, prior to borate $\left(\mathrm{NaBH}_{4}, 0.1 \%\right)$ and cupric $\left(\mathrm{CuSO}_{4}\right.$ in $50 \mathrm{mM}$ ammonium acetate) quenching, strong autofluorescence was identified, which interfered with the detection of real $\beta$-catenin fluorescent signals. The globular structures seen are believed to be lipofuscin and other tissue components, elastin and collagen, including some background signals (believed to be resulting from aldehyde fixative). The emission spectra of natural and fixationinduced fluorescence are quite broad compared to the spectra of proteins of interest which makes it difficult to separate the specific from non-specific fluorescence signals and potentially lead to the noise interference (Kiernan \& Wessendorf, 2001; Monici, 2005). For example, the broad excitation and emission spectra of lipofuscin overlap those of all commonly used fluorophores, which make it difficult or impossible to distinguish between specific labelling and non-specific autofluorescence (Santer et al., 1980; Schnell et al., 1999).

Furthermore, autofluorescence of aldehyde-fixed neural tissue has been shown to obscure neuronal perikarya and fine processes (Clancy \& Cauller, 1998). The probable mechanism of action of $\mathrm{NaBH}_{4}$ in quenching spinal cord background autofluorescence caused by paraformaldehyde fixing of spinal cord tissue is that amines which are released upon cell death could combine with aldehydes in the paraformaldehyde to form Schiff's bases (Davis et al., 2014; Willingham, 1983). The Schiff's bases (crystalline 
in nature) are known to fluoresce, causing the background noise observed in tissue to be excited (Clancy \& Cauller, 1998). Quenching with $\mathrm{NaBH}_{4}$ has previously been shown to neutralise these Schiff's bases through the reduction of amine-aldehyde (such as formaldehyde) compounds into non-fluorescent salts (Clancy \& Cauller, 1998). The action of $\mathrm{CuSO}_{4}$ in quenching spinal cord tissue parenchymal autofluorescence caused by lipofuscin may be through the active radical, $\mathrm{Cu}^{2+}$ by both collisional quenching (in which case electrons are transferred from the lipofuscin to the $\mathrm{Cu}^{2+}$ ) and static quenching (in which a non-fluorescent complex is formed between $\mathrm{Cu}^{2+}$ and lipofuscin) (Schnell et al., 1999). Since fluorescence requires a photon of light to be absorbed by a molecule such as lipofuscin, leading to excitation of electron from its ground state to its excited state thereby releasing energy in the form of another photon, the collisional quenching by $\mathrm{Cu}^{2+}$ would circumvent the emission of its fluorescence.

Our sequential protocol has shown that the $\beta$-catenin was reliably detected and localised in white matter regions of the spinal cord, which has not been previously validated. Therefore, our improved approach for the $\beta$-catenin localisation is highly significant as it unravelled an objective identification and illumination of $\beta$-catenin distribution/detection for future studies related to cellular regeneration/repair post $\mathrm{SCl}$ involving therapeutic agents or nutritional supplements affecting Wnt/ $\beta$-catenin pathway.

\subsection{CONCLUSIONS}

Through appropriate sequential quenching of both lipofuscin and fixative-induced autofluorescence using transcardial hypoperfusion, borate $\left(1 \% \mathrm{NaBH}_{4}\right)$ and cupric ( $1 \mathrm{mM} \mathrm{CuSO}_{4}$ in $50 \mathrm{mM}$ ammonium acetate) treatment, $\beta$-catenin was reliably detected and localised in white matter regions of the spinal cord. This objective approach for the $\beta$-catenin localisation is highly significant as it unravelled an objective identification and illuminate on the pattern of distribution of $\beta$-catenin for researcher focusing on spinal cord repair studies via the Wnt/ $\beta$-catenin pathway following $\mathrm{SCl}$. Thus, we recommend that future studies that investigate the role of $\beta$-catenin in spinal cord repair to perform proper autofluorescence quenching to obtain reliable expression profiles. Following our detection of $\beta$-catenin in the white matter regions of the spinal cord, we encourage studies to investigate the molecular mechanism through which $\beta$-catenin may induce myelin regeneration after $\mathrm{SCl}$.

Acknowledgement: This study is supported by the University of Malaya postgraduate research grant (PG 181-2015B) and the authors are grateful to Medical Laboratory Technologists and Assistant Scientific Officers of the Department of Anatomy and Confocal Microscopy Unit of the University of Malaya for their technical support which are prerequisite to the successful accomplishment of the study.

Author Contributions: DA: performed the experiment, data analysis, writing the manuscript. AAA: study design, supervised the project, and writing the manuscript. JS: study design, supervised the project and financial support.

Conflicts of Interest: The authors declare no conflict of interest.

\section{References}

Ahmad-Annuar, A., Ciani, L., Simeonidis, I., Herreros, J., Fredj, N. B., Rosso, S. B., Hall, A. \& Salinas, P. C. (2006). Signaling across the synapse: a role for Wnt and Dishevelled in presynaptic assembly and neurotransmitter release. Journal of Cell Biology, 174(1), 127-139. https://doi.org/10.1083/icb.200511054

Barker, N., \& Born, M. V. D. (2008). Detection of $\beta$-catenin localization by immunohistochemistry. Methods in Molecular Biology, 468, 91-98. https://doi.org/10.1007/978-1-59745-249-6 7

Ciani, L., \& Salinas, P. C. (2005). WNTs in the vertebrate nervous system: from patterning to neuronal connectivity. Nature Reviews Neuroscience, 6(5), 351-362. https://doi.org/10.1038/nrn1665

Clancy, B., \& Cauller, L. (1998). Reduction of background autofluorescence in brain sections following immersion in sodium borohydride. Journal of Neuroscience Methods, 83(2), 97-102. https://doi.org/10.1016/s0165-0270(98)00066-1

Curinga, G., \& Smith, G. M. (2008). Molecular/genetic manipulation of extrinsic axon guidance factors for CNS repair and regeneration. Experimental Neurology, 209(2), 333-342. https://doi.org/10.1016/i.expneurol.2007.06.026

Cuzzocrea, S., Genovese, T., Mazzon, E., Crisafulli, C., Di Paola, R., Muià, C., Collin, M., Esposito, E., Bramanti, P. \& Thiemermann, C. (2006). Glycogen synthase kinase-3ß inhibition reduces secondary damage in experimental spinal cord trauma. Journal of Pharmacology and Experimental Therapeutics, 318(1), 79-89.

https://doi.org/10.1124/ipet.106.102863 
Davis, A. S., Richter, A., Becker, S., Moyer, J. E., Sandouk, A., Skinner, J., \& Taubenberger, J. K. (2014). Characterizing and diminishing autofluorescence in formalin-fixed paraffin-embedded human respiratory tissue. Journal of Histochemistry and Cytochemistry, 62(6), 405-423. https://doi.org/10.1369/0022155414531549

Deyl, Z., Macek, K., Adam, M., \& Vancikova. (1980). Studies on the chemical nature of elastin fluorescence. Biochimica et Biophysica Acta, 625(2), 248-254. https://doi.org/10.1016/0005-2795(80)90288-3

Dill, J., Wang, H., Zhou, F., \& Li, S. (2008). Inactivation of glycogen synthase kinase 3 promotes axonal growth and recovery in the CNS. Journal of Neuroscience, 28(36), 8914-8928. https://doi.org/10.1523/ineurosci.1178-08.2008

Gage, G. J., Kipke, D. R., \& Shain, W. (2012). Whole animal perfusion fixation for rodents. JoVE (Journal of Visualized Experiments) (65), 3564. https://doi.org/10.3791/3564

Gao, K., \& Zhang, Y. (2018). Effect and molecular mechanism of wnt/ $\beta$-catenin signaling pathway on apoptosis after spinal cord injury. Journal of Surgery, 6(1), 3. https://doi.org/10.13188/2332-4139.1000042

Gao, K., Shen, Z., Yuan, Y., Han, D., Song, C., Guo, Y., \& Mei, X. (2016). Simvastatin inhibits neural cell apoptosis and promotes locomotor recovery via activation of $\mathrm{Wnt} / \beta$-catenin signaling pathway after spinal cord injury. Journal of Neurochemistry, 138(1), 139-149. https://doi.org/10.1111/inc.13382

Gao, K., Wang, Y.S., Yuan, Y.J., Wan, Z.H., Yao, T.C., Li, H.H., Tang, P.F. \& Mei, X.F. (2015). Neuroprotective effect of rapamycin on spinal cord injury via activation of the Wnt/ $\beta$-catenin signaling pathway. Neural Regeneration Research, 10(6), $951-$ 957. https://doi.org/10.4103/1673-5374.158360

Kiernan, J., \& Wessendorf, M. (2001). Autofluorescence: Causes and cures. Toronto Western Research Institute, http://www.uhnresearch.ca/wcif

Liu, Y., Wang, X., Lu, C. C., Kerman, R., Steward, O., Xu, X. M., \& Zou, Y. (2008). Repulsive Wnt signaling inhibits axon regeneration after CNS injury. Journal of Neuroscience, 28(33), 8376-8382. https://doi.org/10.1523/ineurosci.1939$\underline{08.2008}$

Lu, G. B., Niu, F. W., Zhang, Y. C., Du, L., Liang, Z. Y., Gao, Y., Yan, T. Z., Nie, Z. K. \& Gao, K. (2016). Methylprednisolone promotes recovery of neurological function after spinal cord injury: association with Wnt/ $\beta$-catenin signaling pathway activation. Neural Regeneration Research, 11(11), 1816-1823. https://dx.doi.org/10.4103\%2F1673-5374.194753

Ma, W., Yang, J. W., Gao, Y., Luo, T., Cheng, J. R., Wang, D. Y., Guo, J. H. \& Li, L. (2015). Expression pattern of $\beta$-catenin during the development of human fetal spinal cord. Romanian Journal of Morphology and Embryology, 56(2 Suppl), $697-701$.

Monici, M. (2005). Cell and tissue autofluorescence research and diagnostic applications. Biotechnology annual review, 11, 227-256. http://doi.org/10.1016/S1387-2656(05)11007-2

Mosiman, V. L., Patterson, B. K., Canterero, L., \& Goolsby, C. L. (1997). Reducing cellular autofluorescence in flow cytometry: an in situ method. Cytometry, 30(3), 151-156. https://doi.org/10.1002/(SICI)1097-0320(19970615)30:3\%3C151::AIDCYTO6\%3E3.0.CO;2-O

Romijn, H. J., van Uum, J. F., Breedijk, I., Emmering, J., Radu, I., \& Pool, C. W. (1999). Double immunolabeling of neuropeptides in the human hypothalamus as analyzed by confocal laser scanning fluorescence microscopy. Journal of Histochemistry \& Cytochemistry, 47(2), 229-236. https://doi.org/10.1177/002215549904700211

Santer, R., Partanen, M., \& Hervonen, A. (1980). Glyoxylic acid fluorescence and ultrastructural studies of neurones in the coeliac-superior mesenteric ganglion of the aged rat. Cell and Tissue Research, 211(3), 475-485. https://doi.org/10.1007/bf00234401

Schnell, S. A., Staines, W. A., \& Wessendorf, M. W. (1999). Reduction of lipofuscin-like autofluorescence in fluorescently labeled tissue. Journal of Histochemistry \& Cytochemistry, 47(6), 719-730. https://doi.org/10.1177/002215549904700601

Shen, Z., Zhou, Z., Gao, S., Guo, Y., Gao, K., Wang, H., \& Dang, X. (2017). Melatonin inhibits neural cell apoptosis and promotes locomotor recovery via activation of the Wnt/ $\beta$-catenin signaling pathway after spinal cord injury. Neurochemical Research, 42(8), 2336-2343. https://doi.org/10.1007/s11064-017-2251-7

Spitzer, N., Sammons, G. S., \& Price, E. M. (2011). Autofluorescent cells in rat brain can be convincing impostors in green fluorescent reporter studies. Journal of Neuroscience Methods, 197(1), 48-55. https://doi.org/10.1016/j.jneumeth.2011.01.029

Suh, H. I., Min, J., Choi, K. H., Kim, S. W., Kim, K. S., \& Jeon, S. R. (2011). Axonal regeneration effects of Wnt3a-secreting fibroblast transplantation in spinal cord-injured rats. Acta Neurochirurgica, 153(5), 1003-1010. https://doi.org/10.1007/s00701-011-0945-1

Sun, Y., \& Chakrabartty, A. (2016). Cost-effective elimination of lipofuscin fluorescence from formalin-fixed brain tissue by white phosphor light emitting diode array. Biochemistry and Cell Biology, 94(6), 545-550. https://doi.org/10.1139/bcb$\underline{2016-0125}$

Tural Emon, S., Uslu, S., Ilgaz Aydinlar, E., Irban, A., Ince, U., Orakdogen, M., \& Gulec Suyen, G. (2017). Effects of ozone on spinal cord recovery via the wnt/ $\beta$-catenin pathway following spinal cord injury in rats. Turkish Neurosurgery, 27(6), 946951. https://doi.org/10.5137/1019-5149.JTN.17508-16.1

van Amerongen, R., Mikels, A., \& Nusse, R. (2008). Alternative Wnt signaling is initiated by distinct receptors. Science Signaling, 1(35), re9. https://doi.org/10.1126/scisignal.135re9 
Willingham, M. C. (1983). An alternative fixation-processing method for preembedding ultrastructural immunocytochemistry of cytoplasmic antigens: The GBS (glutaraldehyde-borohydride-saponin) procedure. Journal of Histochemistry and Cytochemistry, 31(6), 791-798. https://doi.org/10.1177/31.6.6404984

Yin, Z. S., Zu, B., Chang, J., \& Zhang, H. (2008). Repair effect of Wnt3a protein on the contused adult rat spinal cord. Neurological Research, 30(5), 480-486. https://doi.org/10.1179/174313208X284133

Zhang, Y. K., Huang, Z. J., Liu, S., Liu, Y. P., Song, A. A., \& Song, X. J. (2013). WNT signaling underlies the pathogenesis of neuropathic pain in rodents. Journal of Clinical Investigation, 123(5), 2268-2286. https://doi.org/10.1172/JCI65364 